\title{
Téléprésence, visioconférence ou webconférence : enseignement et apprentissage synchrone et distant
}

\section{Telepresence, videoconferencing or web conferencing: synchronous and remote teaching and learning}

\section{La telepresencia, la videoconferencia o la conferencia web: enseñanza y aprendizaje sincrónico y a distancia}

\section{RESPONSABLES DU NUMÉRO :}

Florian Meyer, professeur

Université de Sherbrooke, Canada

florian.meyer@usherbrooke.ca

Geneviève Lameul, professeure

Université de Rennes 2-CREAD, France

genevieve.lameul@univ-rennes2.fr

Simon Bolduc, doctorant en pédagogie de l'enseignement supérieur

Université de Sherbrooke, Canada

simon.bolduc@usherbrooke.ca

RÉSUMÉ

Alors que le monde traverse une crise sanitaire transformant les rapports humains et les rapports à la formation, ce numéro spécial propose de questionner la formation à distance et plus spécifiquement la téléprésence en enseignement supérieur. Les auteurs offrent des éclairages nouveaux en plaçant au cœur de leurs préoccupations la dimension humaine des activités d'enseignement et apprentissage synchrone et distant. 
At a time when the world is going through a health crisis that is transforming human relations and the relationship to training, this special issue proposes to question distance learning and more specifically telepresence in higher education. The authors offer new insights by placing at the heart of their concerns the human dimension of synchronous and distant teaching and learning activities.

Keywords: videoconferencing, telepresence, web conferencing, higher education

\section{RESUMEN}

En un momento en el que el mundo atraviesa una crisis sanitaria que está transformando las relaciones humanas y en relación con la formación, este número especial se propone reflexionar sobre la enseñanza a distancia y, más concretamente, sobre la telepresencia en la enseñanza superior. Los autores ofrecen nuevas perspectivas al situar, en el centro de sus preocupaciones, la dimensión humana de las actividades de enseñanza y aprendizaje sincrónicas y a distancia.

Palabras clave: videoconferencia, telepresencia, conferencia web, educación superior

Malgré un usage grandissant en enseignement supérieur de dispositifs numériques permettant la création de contextes d'enseignement et d'apprentissage synchrones et distants (visioconférence, téléprésence et webconférence), les études scientifiques relatives aux usages et pratiques, aux conditions favorisant un enseignement et un apprentissage de qualité ou encore aux retombées réelles de ces dispositifs sont peu nombreuses. Certains travaux démontrent toutefois que ces dispositifs technologiques ne sont pas nécessairement adaptés aux multiples besoins de l'enseignement et de l'apprentissage (Alhlak, Ramakrisnan, Hameed et Mohseni, 2012) et qu'ils n'ont pas été conçus pour faciliter la mise en œuvre des nombreuses méthodes et approches pédagogiques (Gillies, 2008; Lawson, Comber, Gage et CullumHanshaw, 2010).

Plusieurs questions émergent : comment agissent les acteurs (formateurs, apprenants ou autres) engagés dans de tels dispositifs? En quoi ces dispositifs transforment-ils l'enseignement et l'apprentissage? Quelles compétences doivent développer les acteurs de la formation? Quels rôles peuvent jouer les conseillers et ingénieurs pédagogiques? Quels sont les enjeux institutionnels propres au déploiement de ce type de dispositifs et comment marquent-ils l'enseignement et l'apprentissage? Quelles sont les conditions qui participent du succès de ces contextes particuliers? Quels enjeux méthodologiques émergent de l'étude de ces contextes?

Alors que la formation à distance s'intègre de plus en plus à l'offre de formation en enseignement supérieur et qu'elle mise sur l'exploitation de dispositifs numériques facilitant la téléprésence, ces questions deviennent centrales. La fermeture généralisée des établissements d'enseignement, provoquée par la 
Ce numéro spécial propose ainsi cinq articles complémentaires offrant des pistes de réponses à quelquesunes de ces questions. Le premier article dresse la table et propose tout d'abord une recension systématique d'écrits traitant de l'usage de la téléprésence en formation. M. Petit, G. Lameul et $\mathrm{J}$. Taschereau nous proposent ainsi différentes définitions de la notion de téléprésence ainsi qu'une distinction entre différents dispositifs de formation misant sur la notion de " téléprésence " et favorisant l'apprentissage à distance. Mettant l'accent plus spécifiquement sur quatre dispositifs technopédagogiques: les salles de visioconférence immersives, les robots de téléprésence, la réalité virtuelle et les hologrammes, Petit et ses collègues dressent un portrait d'utilisations potentielles de la téléprésence en formation.

Inscrits dans la rubrique "Recherche ", les deux articles suivants entrent plus spécifiquement dans des études propres à des dispositifs distincts. Les expérimentations technopédagogiques dont ces articles traitent émanent d'initiatives proposées dans le cadre de l'appel à projets dit "campus numérique " (Thibault, 2007) du ministère de l'enseignement supérieur et de la recherche français. Tout d'abord, B. Savarieau et $\mathrm{H}$. Daguet présentent les résultats d'une recherche liée au projet "Forse », un campus numérique de l'Université de Rouen. Dans cet article, les auteurs s'intéressent à l'usage des classes virtuelles synchrones et se questionnent à savoir si ce dispositif modifie la professionnalité enseignante et délimitent les possibles transformations qui en résultent. Cet article présente l'originalité de s'intéresser à l'expérience des enseignants en formation à distance (plus rarement étudiée que l'expérience des étudiants), en nous montrant leur activité en classe virtuelle dans l'intimité de leur espace personnel, en donnant à voir comment cette porosité des espaces personnel/professionnel vient questionner leur métier et comment la relation à distance réinterroge la place du corps parlant (Jorro, 2006) de l'enseignant.

Ensuite, S. Bolduc, M. Petit, F. Meyer, D. Bédard et R. Challah présentent les résultats d'une recherche menée au sein du projet TOPIC (Téléprésence comme OPportunité d'Innovation dans la Conception de formation) qui réunissait l'Université de Sherbrooke au Canada (UdeS) et l'Université Bretagne Loire en France (UBL) impliquée dans le déploiement du projet «UEB C@ampus ». Les auteurs s'intéressent plus largement aux usages des dispositifs technopédagogiques de type visioconférence dans les deux universités partenaires. Pour eux, la question des usages de ces dispositifs se rapporte à celle de la formation des formateurs et ils s'intéressent donc aux usages actuels, au niveau d'aisance et aux attentes de formation de ces derniers.

Ainsi, nous retrouvons dans l'article de Savarieau et Daguet un intérêt pour une meilleure compréhension des pratiques pédagogiques instrumentées en contexte de classe virtuelle, tandis que Bolduc et ses collègues s'intéressent aux dispositifs de formation continue à mettre en place pour répondre aux besoins et aux attentes des formateurs qui font usage de ces dispositifs. Néanmoins, tous les deux traitent de la transformation des rôles des différents acteurs qu'engendre l'utilisation de ces nouveaux dispositifs technopédagogiques, dont les enseignants et les institutions.

Les deux derniers articles ouvrent la réflexion en offrant une lecture plus théorique de la notion de téléprésence selon le point de vue de deux experts. Ainsi, dans la rubrique " Notes de lecture », M. Petit propose une analyse synthèse de l'ouvrage collectif La téléprésence en formation dirigé par Jean-Luc Rinaudo en 2018. II en fait partager sa lecture en pointant des éléments qui font écho à certains écrits de ce numéro : importance de préciser les mots qui gravitent autour de "téléprésence »; interrogation du rapport au corps; questionnement des compétences numériques, etc.

Et, finalement, dans la rubrique " entretien », S. Androwkha propose une entrevue avec Annie Jézégou. Par ses questions précises, Androwkha propose à Jézégou de discuter de la présence à distance en e-Formation et notamment des affordances socionumériques inhérentes aux contextes de formation misant sur des outils numériques d'enseignement et apprentissage synchrones et distants. Ainsi, argumentant sur le rôle essentiel de l'affordance « perçue » des utilisateurs et l'agentivité des acteurs de la formation pour activer les possibilités de communication à distance de ces artéfacts, Jézégou propose 
la formation pour activer les possibilités de communication à distance de ces artéfacts, Jézégou propose un regard sur les conditions pour générer une dynamique relationnelle et médiatisée porteuse d'un sentiment de présence à distance.

Ce numéro spécial prend une actualité tout à fait particulière dans le contexte de crise sanitaire qui ébranle le monde entier en ce début d'année 2020 - qui bouleverse notamment le système d'éducation auquel il s'intéresse. Au-delà des éclairages complémentaires qu'apporte ce numéro aux travaux existants, il laisse bien entrevoir la nécessité de dépasser les études exploratoires pour s'engager dans des recherches multidisciplinaires d'envergure qui permettront de mieux cerner le potentiel de la relation à distance instrumentée. Cette tragique catastrophe humanitaire que nous subissons constitue une alerte et une invitation particulière à reprendre les réflexions de G. Jacquinot qui dès 1993 attirait notre attention sur la nécessité d'apprivoiser la distance et de questionner les différentes formes de présence qu'elle est susceptible de générer. L'étude des usages " apprivoisés » des dispositifs techniques de téléprésence, visioconférence ou webconférence peut documenter utilement les processus d'hybridation et requestionner la dimension humaine des activités d'enseignement et apprentissage synchrone et distant.

\section{Liste de références}

Alhlak, B.A., Ramakrisnan, P., Hameed, Z. S. et Mohseni, H.R. (2012). Video conference: integrated tool for identifying CSF in education development in UiTM. Procedia-Social and Behavioral Sciences, 2012(67), 102-113.

Jacquinot, G. (1993). Apprivoiser la distance et supprimer l'absence? ou les défis de la formation à distance, Revue pédagogique, 1993(102), 55-67

Jorro, A. (2006). L'agir professionnel de l'enseignant. Présenté à Séminaire du centre de Recherche sur la formation, CNAM. Consulté à l'adresse http://halshs.archives-ouvertes.fr/halshs-00195900/f

Gillies, D. (2008). Student perspectives on videoconferencing in teacher education at a distance. Distance education, 29(1), 107-118.

Lawson, T., Comber, C., Gage, J. et Cullum-Hanshaw, A. (2010) Images of the future for education? Videoconferencing: a literature review. Technology, pedagogy and education, 19(3), 295-314.

Rinaudo, J.-L. (2018). La téléprésence en formation. Londres, Angleterre : les éditions ISTE.

Thibault, F. (2007). Campus numérique; archéologie d'une initiative ministérielle, Études de communication, Numéro spécial, 17-48.

\section{Articles du numéro :}

1. «La téléprésence en formation - Définitions et catégories d'utilisation issues d'une recension systématique des écrits » par M. Petit, G. Lameul et J. Taschereau.

2. «L'intégration de la classe virtuelle synchrone à l'université, un levier de transformation de la professionnalité enseignante? » par B. Savarieau et H. Daguet.

3. «Se former à l'usage des dispositifs de téléprésence, visioconférence et webconférence en enseignement supérieur » par S. Bolduc, M. Petit, F. Meyer, D. Bédard et R. Challah.

4. «La présence à distance en e-Formation - Entretien avec Annie Jézégou » par S. Androwkha.

5. « Recension — Rinaudo, J.-L. (2018). La téléprésence en formation. Londres : Éditions ISTE » par M. Petit. 